\title{
Compatibility of Polyether Sulfone and Polyimide Derived from Isomerization of Polyisoimide. An Approach to In-Situ Generated Rigid-Rod Molecular Composite
}

\author{
Amane Mochizuki, Kazuo Yamada, Mitsuru Ueda \\ and Rikio YoKota* \\ Department of Materials Science and Engineering, Faculty of Engineering, \\ Yamagata University, Yonezawa 992, Japan \\ * Institute of Space and Astronautical Science, \\ Komaba, Meguro-ku, Tokyo 153, Japan \\ (Received October 2, 1996)
}

\begin{abstract}
An approach to in-situ generated rigid-rod molecular composites (RRMC) was investigated. This method requires a coil-like aromatic polyisoimide (PII) which soluble and compatible with an amorphous matrix polymer and can undergoes thermal isomerization to the corresponding rigid-rod polyimide (PI) in the solid composite state. PII was prepared by the ring-opening polyaddition of pyromellitic dianhydride (PMDA) and 2,2',6,6'-tetramethylbenzidine (TMBZ) followed by treatment with trifluoroacetic anhydride-triethylamine in NMP. A miscible solution blend of PII and poly(oxy-1,4phenylenesulfonyl-1,4-phenylene) (PES) was obtained. The compatibility of blend films was investigated by transmission electron microscopy (TEM). The diameter of PI domain for the PI/PES, 4/96 was found to be approximately $0.2 \mu \mathrm{m}$, and the formation of an interpenetrating structure was confirmed by the elemental analysis on the domain part. Transparent composite films were obtained at PI content of 2 to $10 \mathrm{wt} \%$.

KEY WORDS Rigid-Rod Molecular Composite (RRMC) / Polyisoimide / Polyethersulfone / Compatibility /
\end{abstract}

Aromatic poly(ether sulfone)s for instance poly(oxy1,4-phenylenesulfonyl-1,4-phenylene) (PES) ${ }^{1}$ and poly(oxy-1,4-phenyleneisopropyridene-1,4-phenyleneoxy1,4-phenylenesulfonyl-1,4-phenylene)(PSF) ${ }^{2}$ have been developed for use as high performance engineering thermoplastics. However, considerable attention has been directed to modifying these polymers because it is difficult for one component of poly(ether sulfone)s to have all the required properties such as thermal, chemical, and mechanical properties, and a number of novel polymer blends based on poly(ether sulfone)s have been reported. ${ }^{3-5}$

One approach to obtain such properties is blending a rod-like polymer system with a flexible polymer to provide enhanced tensile properties and greater toughness. However, the intrinsic tendency toward phase separation between rodlike and flexible polymers invariably leads to phase-separated morphologies. Thus, various parameters affecting the phase separation of rodlike/flexible polymer mixtures and morphologies of resulting blend film have been investigated.

Yokota et al. ${ }^{6}$ investigated that solution blending of rigid poly ( $p$-phenylene biphenyltetracarboxyimide) (BPDA-p-PDA) with flexible poly(4,4'-diphenylether biphenyltetracarboxyimide) (BPDA-ODA) and concluded that the rigid PI (BPDA- $p$-PDA) molecules are oriented and dispersed at the molecular level in the flexible PI (BPDA-ODA) matrix. Morikawa et al. ${ }^{7}$ reported a new class of polyimide-silica hybrid materials with high silica content and flexible polyimide-silica film containing up to $70 \mathrm{wt} \%$ of silica prepared by sol-gel reaction of tetraethoxysilane (TEOS) in the polyamic acid solution. 'In situ polycondensation' was suggested by Ogata and Sanui. ${ }^{8}$ The formation of polyamic acid (pyromellitic dianhydride (PMDA)-ODA) in poly(vinyl- pyridine-co-styrlene) (PVPy-St) solutions was investigated and the tensile strength of these components was found higher than that of either individual polymer. ${ }^{9}$ Wallace et al. ${ }^{10}$ proposed the approach of in-situ generated rigid-rod molecular composites (RRMC) using coil-like PII derived from 3,3',5,5'-tetramethylbenzidine and PMDA and commercial matrix systems, polyarylsulfone (Radel) and a polysulfone (Udel). However, phase separated cloudy films were obtained from the solution of matrix polymer and PII, and no other study has been reported on blend films.

Efforts to increase the solubility of rigid-rod polyimides include the use of non-coplanar biphenyl moieties. Rigid-rod polyimides derived from $2,2^{\prime}, 6,6^{\prime}$-tetramethylbenzidine (TMBZ) have been reported to display high glass transition temperature $\left(T_{\mathrm{g}}\right)$ and excellent thermal stability due to the increased rotational barrier of the PI back bone. ${ }^{11}$ These PIs are soluble in $m$-cresol or 1,1,2,2tetrachloroethane. However, PI prepared from PMDA and TMBZ which has the most rigid-structure is still insoluble in common organic solvent.

In the preceding paper, ${ }^{12}$ we reported the preparation and properties of polyisoimide (PII) as a PI-precursor, and found that PII has higher solubility and lower $T_{\mathrm{g}}$ than that of corresponding PI and is easily converted to PI without elimination of volatile compounds. Therefore, PII is of considerable interest as a candidate for a rigid-rod PI-precursor.

In this paper, PII(a) (PMDA/TMBZ) and PES were used as the rigid-rod PI-precursor and flexible matrix polymer, respectively. We report the synthesis of PII and compatibility of the PES and PI which is induced by thermal isomerization of PII. 


\section{EXPERIMENTAL}

\section{Materials}

$N$-methyl-2-pyrrolidone (NMP), $N, N$-dimethylacetamide (DMAc) and triethylamine (TEA) were purified by vacuum distillation. Pyromellitic dianhydride (PMDA) was purified by recrystallization from acetic anhydride. Poly(oxy-1,4-phenylenesulfonyl-1,4-phenylene) (PES: Victrex 4100p) was obtained from Sumitomo Chemical Co., Ltd. Other reagents and solvents were obtained commercially and used as received.

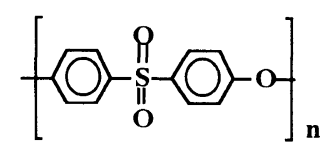

PES

\section{Synthesis of 2,2',6,6'-Tetramethylhydrazobenzene}

5-Nitro-1,3-xylene $(10.0 \mathrm{~g}, 41.6 \mathrm{mmol})$ and zinc dust $(25.0 \mathrm{~g})$ were dissolved in ethanol $(40 \mathrm{~mL})$. To this solution was added with stirring $30 \mathrm{wt} \%$ of aqueous sodium hydroxide solution $(50 \mathrm{~mL})$. The mixture was heated at reflux for $4 \mathrm{~h}$, cooled to room temperature and filtered off and the filtrate was poured into $30 \mathrm{wt} \%$ acetic acid aqueous solution. The precipitate was collected by filtration, washed with water and dried in vacuo. Recrystallization from a petroleum-ether produced pale yellow needles. $\mathrm{mp} 124-125^{\circ} \mathrm{C}$ (lit. ${ }^{13} 122-124^{\circ} \mathrm{C}$ ); IR (KBr): v $3300 \mathrm{~cm}^{-1}(\mathrm{~N}-\mathrm{H}), 2900 \mathrm{~cm}^{-1}\left(-\mathrm{CH}_{3}\right)$; Anal. Calcd for $\mathrm{C}_{16} \mathrm{H}_{20} \mathrm{~N}_{2}: \mathrm{C}, 79.96 \% ; \mathrm{H}, 8.39 \% ; \mathrm{N}, 11.66 \%$. Found: $\mathrm{C}, 80.04 \% ; \mathrm{H}, 8.39 \%$;, $11.54 \%$.

\section{Synthesis of 2,2',6, $6^{\prime}$-Tetramethylbenzidine (TMBZ)}

$3,3^{\prime}, 5,5^{\prime}$-Tetramethylhydrazobenzene $(2.46 \mathrm{~g}, 10.0$ mmol) was added to $3 N$-hydrochloric acid aqueous solution $(100 \mathrm{~mL})$. The mixture was heated at reflux for $4 \mathrm{~h}$, filtered off and the filtrate was refluxed additional $30 \mathrm{~min}$. The reaction mixture was cooled to room temperature and treated with $20 \mathrm{wt} \%$ sodium hydroxide aqueous solution until cloudiness appeared and then with a saturated solution of sodium acetate until precipitation was complete. This solution was extracted with three $100 \mathrm{~mL}$ portion of ether. The red ether solution was dried over magnesium sulfate and concentrated. The ether was removed and replaced by $20 \mathrm{~mL}$ benzene. The hot benzene solution was treated with $40 \mathrm{~mL}$ petroleum ether and the resulting solution was cooled. The buff colored needles were collected by filtration and dried. The residue was recrystallized from $50 \%$ ethanol to give pale yellow needles. mp $167-169^{\circ} \mathrm{C}$ (lit. $\left.{ }^{13} 167-168^{\circ} \mathrm{C}\right)$; IR (K Br) $v$ $3300 \mathrm{~cm}^{-1}(\mathrm{~N}-\mathrm{H}), \quad v \quad 3300 \mathrm{~cm}^{-1}(\mathrm{~N}-\mathrm{H}), 2900 \mathrm{~cm}^{-1}$ $\left(-\mathrm{CH}_{3}\right)$; Anal. Caled for $\mathrm{C}_{16} \mathrm{H}_{20} \mathrm{~N}_{2}: \mathrm{C}, 79.96 \% ; \mathrm{H}$, $8.39 \%$; N, $11.66 \%$. Found: C, $79.82 \% ; \mathrm{H}, 8.42 \%$; N, $11.38 \%$.

\section{Polyisoimide (PII)(a) Synthesis}

A solution of TMBZ $(1.20 \mathrm{~g}, 5.0 \mathrm{mmol})$ in NMP $(19.2 \mathrm{~mL})$ was cooled in an ice-water bath. To this solution was added with stirring PMDA $(1.09 \mathrm{~g}, 5.0$ $\mathrm{mmol})$. The mixture was stirred at room temperature for $19 \mathrm{~h}$. The resulting viscous solution was diluted with NMP $(90.0 \mathrm{~mL})$ and TEA $(1.40 \mathrm{~mL}, 10.0 \mathrm{mmol})$ was added dropwise with stirring. The reaction mixture was cooled with an ice-water bath and trifluoroacetic anhydride $(2.10 \mathrm{~mL}, 15.0 \mathrm{mmol})$ was added dropwise with stirring. The mixture was stirred at room temperature for $4 \mathrm{~h}$ and poured into 2-propanol $(1000 \mathrm{~mL})$. The polymer precipitated was filtered off and dried in vacuo at $40^{\circ} \mathrm{C}$. The yield was $3.07 \mathrm{~g}(96 \%)$. IR $(\mathrm{KBr}): v$ $1800 \mathrm{~cm}^{-1}(\mathrm{C}=\mathrm{O}), 920 \mathrm{~cm}^{-1}(\mathrm{C}-\mathrm{O})$. Anal. Calcd for $\left(\mathrm{C}_{26} \mathrm{H}_{20} \mathrm{~N}_{2} \mathrm{O}_{4} \cdot \mathrm{H}_{2} \mathrm{O}\right)_{n}: \mathrm{C}, 70.58 \% ; \mathrm{H}, 5.01 \% ; \mathrm{N}, 6.33 \%$. Found: C, $70.64 \%$; $4.81 \%$; N, $6.30 \%$.

\section{Preparation of Blend Film}

A solution of PII and PES was made by dissolving the polymers in DMAc to afford a $20 \mathrm{wt} \%$ solution. Films cast on glass plates were dried at $80^{\circ} \mathrm{C}$ for $10 \mathrm{~min}$ and dried in vacuo at $200^{\circ} \mathrm{C}$ for $3 \mathrm{~h}$. The thickness of the polymer film was $25 \mu \mathrm{m}$.

\section{Measurement}

The infrared spectra were recorded on a Hitachi I-5020 FT-IR spectrophotometer. Thermal analysis was performed on a Seiko SSS 5000-TG/DTA 200 at a heating rate of $10^{\circ} \mathrm{C} \mathrm{min}^{-1}$ for TG and a Seiko SSS 5000 DSC220 at a heating rate of $10^{\circ} \mathrm{C} \mathrm{min}^{-1}$ for differential scanning calorimetry (DSC) under nitrogen. Molecular weights were determined by gel permeation chromatography (GPC) with polystyrene calibration using JASCO HPLC system equipped with Shodex KD-80M column at $40^{\circ} \mathrm{C}$ in DMF containing $10 \mathrm{mM}$ of $\mathrm{LiBr}$. Transmittance of blend films was determined by BHPS polarization microscopy (Olympus Co., Ltd.). The morphologies of cross-sectional view of blend films were studied by transmission electron microscopy (TEM) (Hitachi, H-800 at $100 \mathrm{kV}$ of accelerating voltage) and energy-dispersive $\mathrm{X}$-ray analysis (EDX) (Kevex Delta IV). Number average diameter of domain size was determined from the TEM photographs at the hundred samples whose values were averaged.

\section{RESULTS AND DISCUSSION}

\section{Synthesis of Polyisoimide PII(a)}

The selection of a rigid-rod polyimide (PI) precursor is very important to make RRMC consisting of rigid-rod PI and flexible polyether sulfone (PES), because the key principle is the thermally induced isomerization of a coil-like precursor to the respective rigid rod within the matrix polymer. The rigid-rod precursor and matrix polymer material must be compatible and soluble in a common organic solvent to produce homogeneous solution.

Introducing a noncoplanar biphenyl moiety into the main polymer chain is special interest. ${ }^{11,14,15}$ The substituent at the 2,2'-position on the biphenyl moiety forces the two phenyl rings into a non-coplanar conformation. This structure would not have crystal packing, thus increasing solubility.

Therefore, we selected the polyisoimide (PII)(a) from pyromellitic dianhydride (PMDA) and 2,2',6,6'-tetramethylbenzidine (TMBZ) as a candidate of rigid-rod precursor.

TMBZ was prepared in two steps as reported procedure. ${ }^{13}$ 5-Nitro- $m$-xylene was reacted with zinc in eth- 
anolic sodium hydroxide to give $3,3^{\prime}, 5,5^{\prime}$-tetramethylhydrazobenzene, which subsequently underwent benzidine rearrangement to afford TMBZ (eq 1). PII(a) was prepared by ring-opening polyaddition of PMDA and TMBZ, followed by treatment with trifluoroacetic anhydride-TEA in NMP (eq 2).

The polymer was obtained as a pale yellow powder and confirmed to corresponding PII(a) by infrared spectroscopy and elemental analysis. The IR spectra exhibited characteristic absorption at $1800 \mathrm{~cm}^{-1}$ due to the isoimide carbonyl. Elemental analysis supported the formation of the expected polymer. Imide content was determined by comparing the absorption of imide $\left(1380 \mathrm{~cm}^{-1}\right)$ and the internal standard peak at $1490 \mathrm{~cm}^{-1}$ by IR spectroscopy ${ }^{16}$ was less than $5 \%$.

Chan et al. ${ }^{11}$ reported PI prepared from PMDA and TMBZ is insoluble in organic solvents, such as 1,1,2,2tetrachloroethane, $m$-cresol, or NMP. However, PII(a) prepared in this investigation was soluble in aprotic polar solvents, such as NMP, DMF, and DMAc. A transparent film was cast from the DMAc solution. The molecular weight of the polymer was determined by GPC. The GPC trace was unimodal with polydispersity of 1.8 . The chromatogram indicated relative $\bar{M}_{n}$ and $\bar{M}_{w}$ to be 53,000 and 94,000 , respectively, for standard polystyrene.<smiles>Cc1cc(C)cc(NNc2cc(C)cc(C)c2)c1</smiles><smiles>Cc1cc(N)cc(C)c1-c1c(C)cc(N)cc1C</smiles>

TMBZ

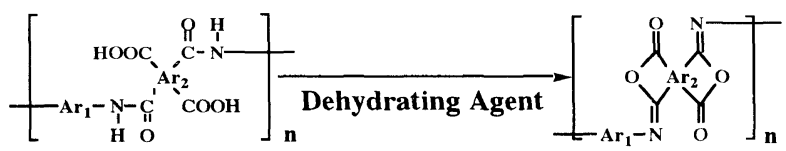

Polyamic acid

Polyisoimide (PII) (a)

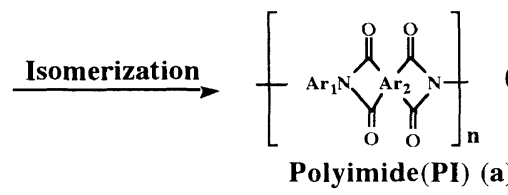

$\mathbf{A r}_{1}:$

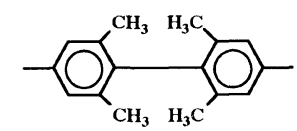

TMBZ
$\mathbf{A r}_{2}$ :

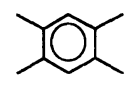

PMPA

\section{Thermal Properties of PII}

$T_{\mathrm{g}}$ of PII(a) would be expected to be different from that of the corresponding $\operatorname{PI}(\mathbf{a})$ due to the coil like structure, analogous to the imide form. ${ }^{12}$ To study the thermal properties of PII(a), DSC measurement was performed in powder state. PII(a) shows no transition peak till $400^{\circ} \mathrm{C}$, in the 1 st and 2 nd heating processes.

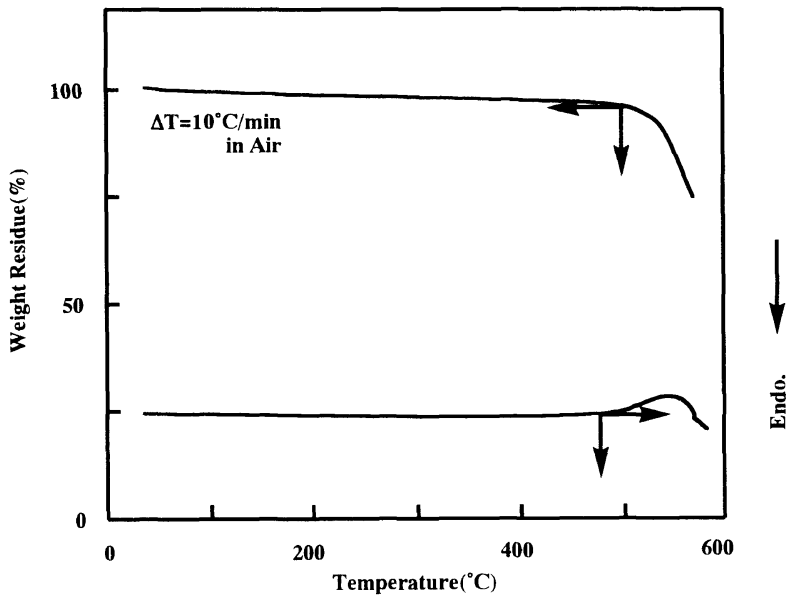

Figure 1. TG/DTA curves of PII(a).

The IR spectra of the polymer showed isoimide and imide carbonyl absorptions, even after the 2nd heating process. TGA/DTA curves of PII(a) are shown in Figure 1. The polymer showed $10 \%$ weight loss at $510^{\circ} \mathrm{C}$ in air. Typically, these aromatic poly(pyromellitimides) with the $p$-catenated aromatic diamine display very high softening temperatures and limited solubility in aprotic and protic solvents. PII(a) has higher thermal stability in addition to excellent solubility in organic solvents such as NMP or DMAc and expected to isomerize to the corresponding rigid-rod geometry by appropriate thermal treatment involving temperature and catalyst.

\section{Compatibility of PII and PES}

Compatibility of binary polymer blend was studied. PES is soluble in similar aprotic polar solvents such as NMP or DMAc, which dissolve PII(a). Therefore, the compatibility between PII(a) and PES was first studied for the solution blend in DMAc with the composition from 2/98 to 30/70 for PII/PES, and 20/80 for polymer/ solvent on a weight basis. All compositions gave homogeneous solutions even in $30 \mathrm{wt} \%$ of PII. Subsequently, the composite films were prepared by casting onto glass plates and drying at $80^{\circ} \mathrm{C}$ for $10 \mathrm{~min}$, then residual solvent was removed by heating at $200^{\circ} \mathrm{C}$ for $3 \mathrm{~h}$ in vacuo.

After drying at $200^{\circ} \mathrm{C}$, the IR spectrum of the composite film (PES $=70 \mathrm{wt} \%$ ) showed strong imide carbonyl absorptions at 1780 and $1720 \mathrm{~cm}^{-1}$, while the characteristic absorption bands of the isoimide group at 1800 $\mathrm{cm}^{-1}$ and $920 \mathrm{~cm}^{-1}$ were greatly reduced in intensity. This indicates that PII in the composite film is gradually converted to the corresponding PI(a) during vacuum drying of the film. Previously, we showed the thermal isomerization reaction of PII to the corresponding PI to be accelerated by a catalytic amount of amine or acid, ${ }^{12}$ and this feature was applied to developing a photosensitive PI precursor. ${ }^{17}$ Although DMAc is a weak basic solvent, it is considered to act not only as a solvent, but also as a base catalyst. Therefore, the complete isoimideimide conversion was induced during film formation.

Films with PI(a) content bellow $10 \mathrm{wt} \%$ exhibited highly transparency, while blend films PI/PES 20/80, and 30/70 were opaque and translucent (Table I). DSC measurement was also performed to investigate the compatibility of these films and the results are shown in 


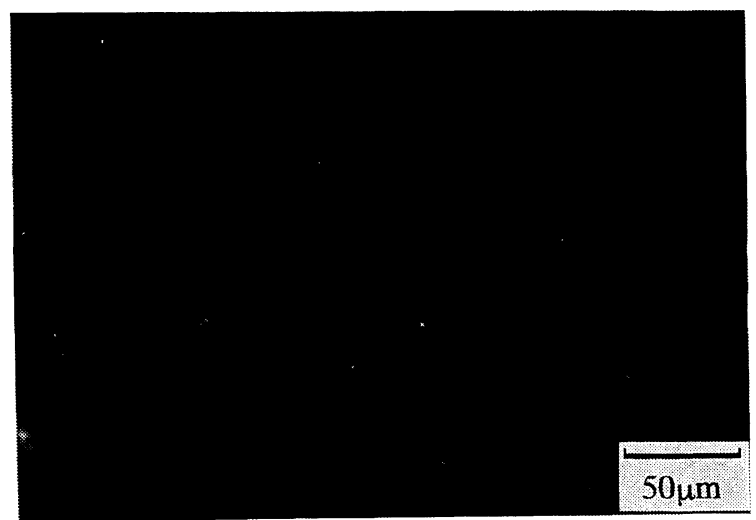

Figure 2(1).

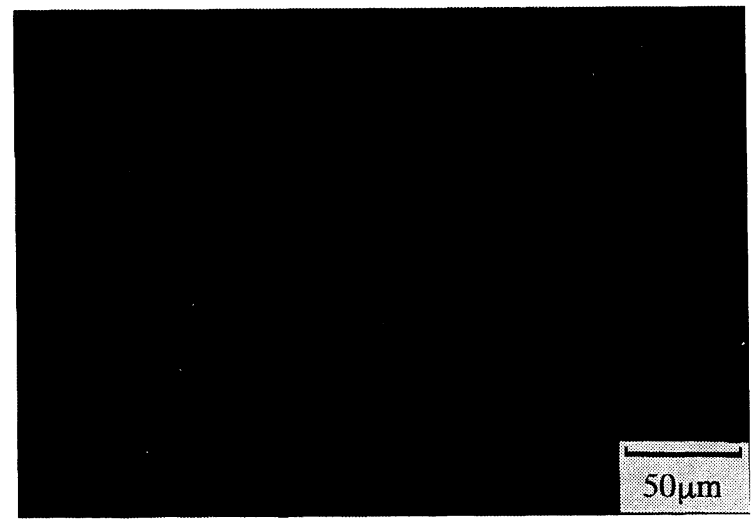

Figure 2(2).

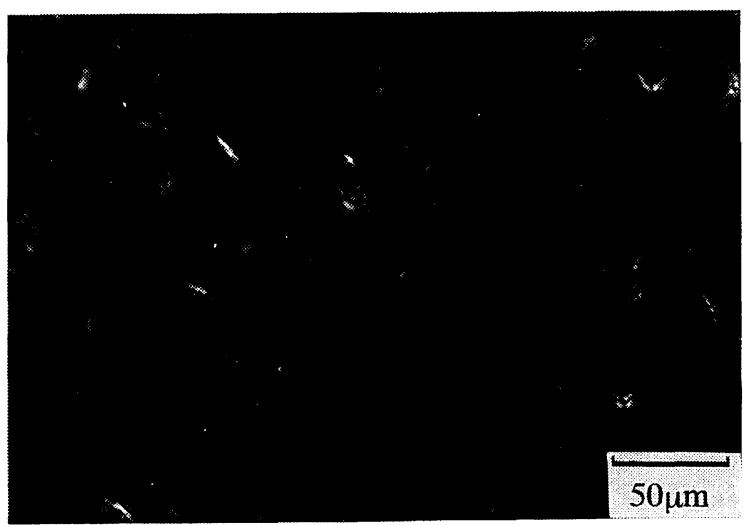

Figure 2(3)

Table I. Properties of blend films

\begin{tabular}{ccc}
\hline $\mathrm{RII} / \mathrm{wt} \%$ & $T_{\mathrm{g}} /{ }^{\circ} \mathrm{C}^{\mathrm{a}}$ & Appearance $^{\mathrm{b}}$ \\
\hline 0 & 218 & $\mathrm{~T}$ \\
2 & 224 & $\mathrm{~T}$ \\
4 & 224 & $\mathrm{~T}$ \\
10 & 226 & $\mathrm{~T}$ \\
20 & 233 & $\mathrm{O}$ \\
30 & 227 & $\mathrm{O}$ \\
100 & $-\mathrm{c}$ & $\mathrm{T}$
\end{tabular}

a Temperature at inflection point in the DSC thermograms with heating rate of $10^{\circ} \mathrm{C} \mathrm{min}^{-1}$ in nitrogen. ${ }^{b} T$, transparent; $\mathrm{O}$, opaque ${ }^{\mathrm{c}}$ No $T_{\mathrm{g}}$ could be detected.

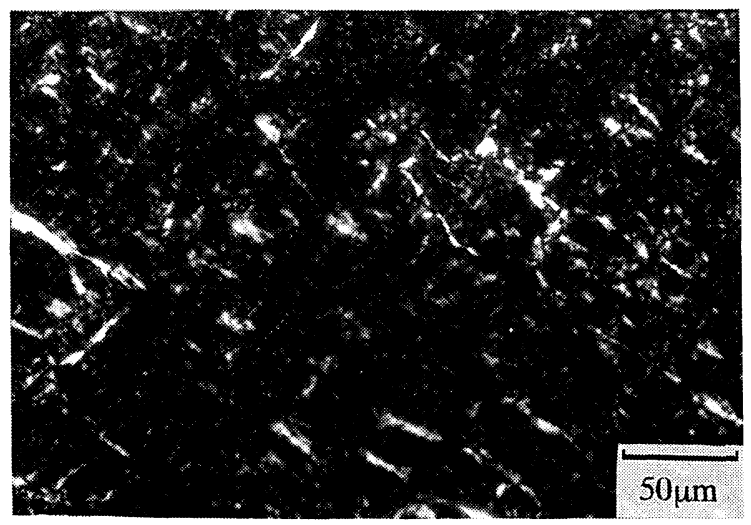

Figure 2(4).

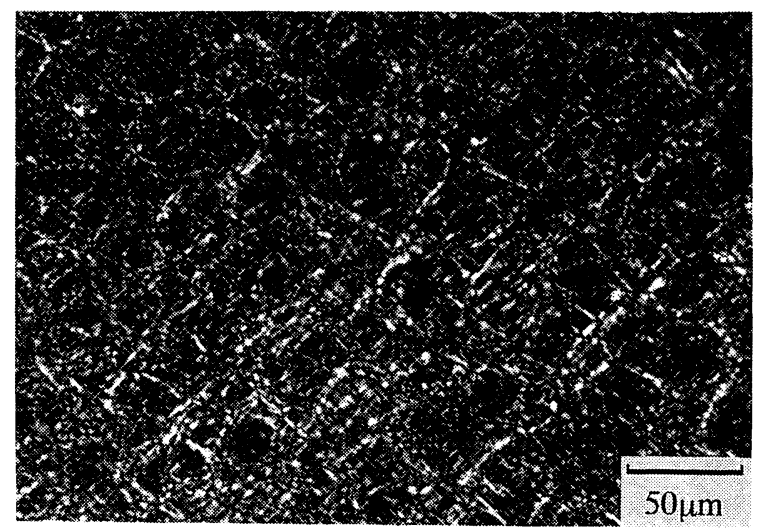

Figure 2(5).

Figure 2. Polarized optical micrographs of blend films observed at room temperature (between crossed polarizers). (1), PI/PES 2/98; (2), PI/PES 4/96; (3), PI/PES 10/90; (4), PI/PES 20/80; (5), PI/PES 30/70.

Table I. All the films exhibited endothermic peaks and these peaks slightly rising with increasing $\mathrm{PI}(\mathbf{a})$ contents. The above observation was not sufficient to indicate the compatibility of PI and PES, because $T_{\mathrm{g}}$ of $\mathrm{PI}(\mathbf{a})$ is too high to detect by DSC measurement. Thus, to investigate the compatibility of these components in more detail, the composite films were observed using a polarized optical microscope. Figures 2(1) - (5) show photographs of the blend films observed under polarized microscope. The blend films with less than $10 \mathrm{wt} \% \mathrm{PI}(\mathbf{a})$ content exhibited dark view indicate that PI(a) in these films considered to be well dispersed in PES matrix in a fine particle form with a diameter of less than $400 \mathrm{~nm}$. However, birefringent light was observed in blend films with more than $20 \mathrm{wt} \%$ PI content due to thermallyinduced phase separation during vaporization of solvent. Composite films with less than $10 \mathrm{wt} \% \mathrm{PI}(\mathbf{a})$ thus have a homogeneous phase.

\section{Morphologies of Blend Films}

A molecular composite is defined as rigid-rod molecules molecularly dispersed in a matrix with a flexible coil-like structures and the rod-like molecules act as the reinforcing elements. Thus far, extensive studies on compatibility of the blend films prepared from polyimide and PES have not been done, although experimental results have been reported. Thus, in this investigation, 

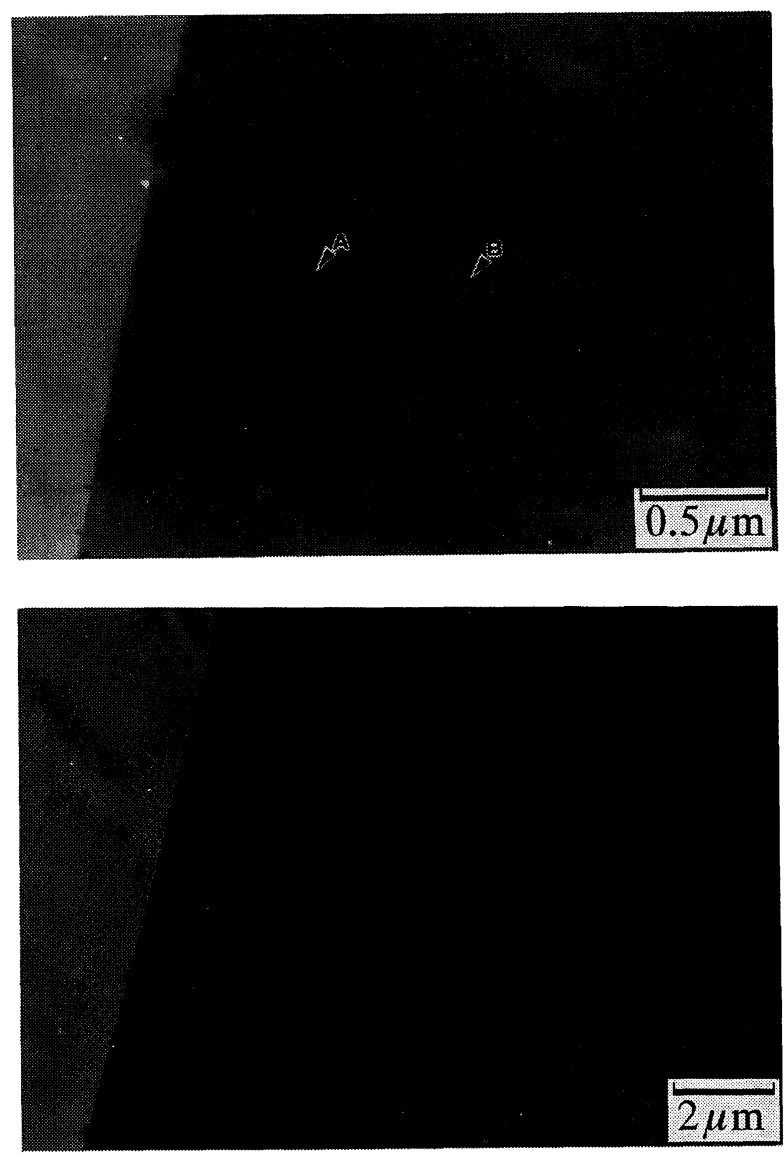

Figure 3(1)
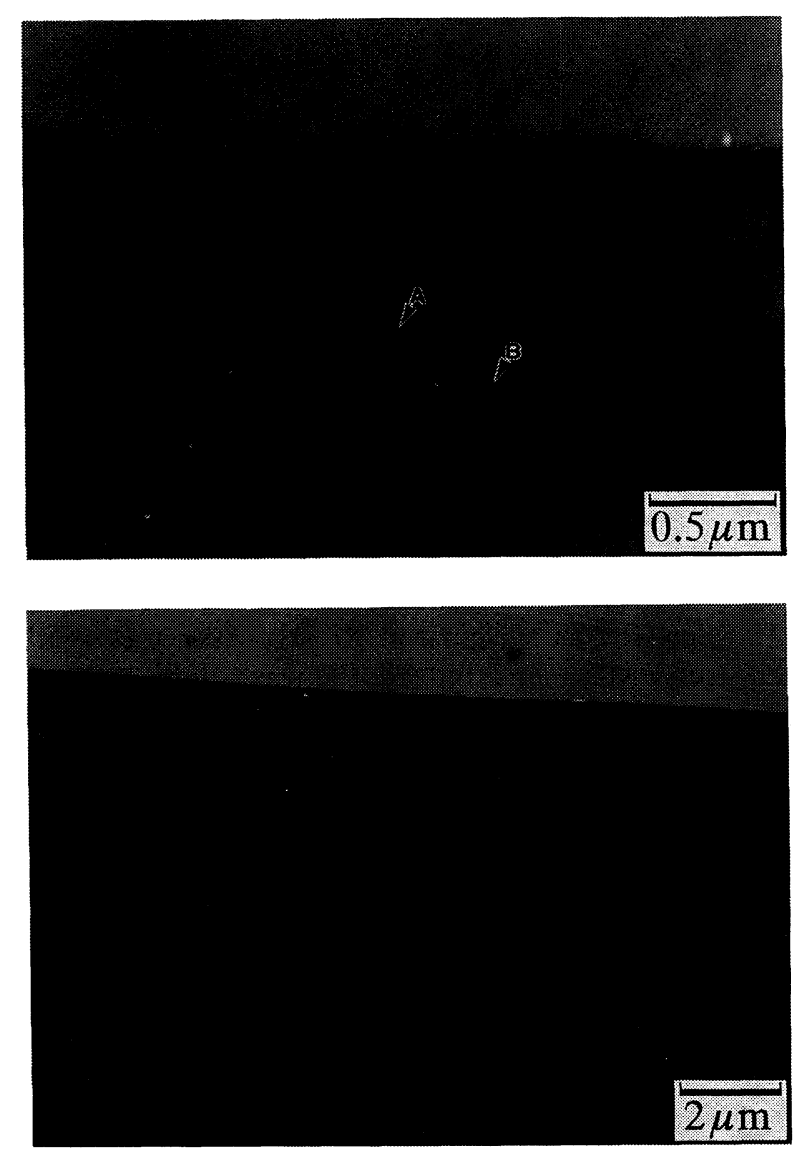

Figure 3(2).
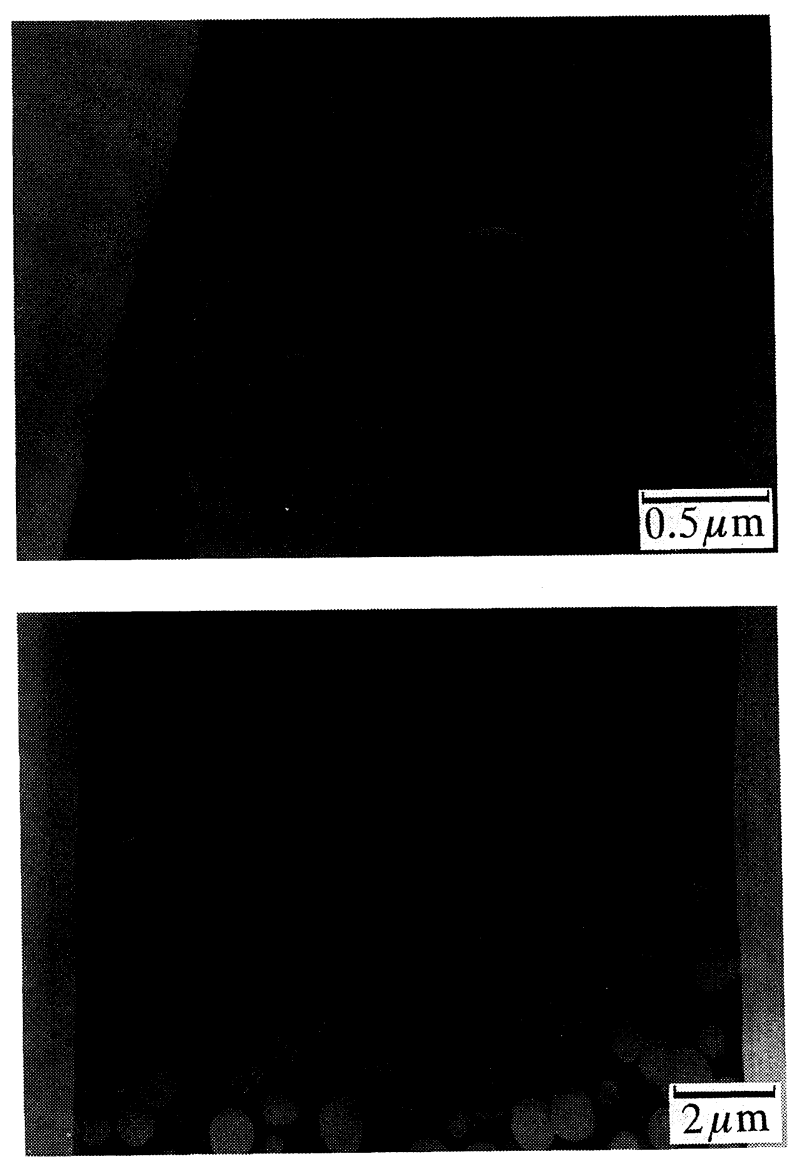

Figure 3(3).

Figure 3. TEM photographs of blend film (1), (PI/PES, 4/96); (2), PI/PES 10/90; (3), PI/PES 30/70.

the morphologies of composite films were investigated by transmission electron microscopy (TEM) to investigate the molecular dispersity of the rigid-rod PI in the PES as a matrix polymer. Figures 3(1)-(3) show TEM micrographs of blend films. All the films were heterogeneous and had two phase structures with the PI domain dispersed in the matrix, whereas the blend films containing less than $10 \mathrm{wt} \% \mathrm{PI}(\mathbf{a})$ were transparent under a polarized microscope as shown in Figure 2 (3). The average diameter of the domains are gradually increased with PI(a).

To clarify the composition of blend films in more detail, the number average diameter of domains was measured. Figure 4 shows the relationship between the PI(a) content and average diameter of domains which become gradually larger with increasing PI. The domain size of the composite film containing $4 \mathrm{wt} \%$ of $\mathrm{PI}(\mathbf{a})$ was found to be approximately $0.2 \mu \mathrm{m}$. The domain is thus PI(a).

The domain and matrix in these TEM photographs were analyzed by an energy-dispersive X-ray analyzer (EDX) to clarify the component of each phase. The results are shown in Figures 5-8. Figures 5 and 7 show sulfur atoms derived from PES to be present in all matrix regions (part A) and this is confirmed in Figures 3(1) and (2). In Figure 8, trace sulfur existence is slightly observed in the domain part (part B) in Figure 3(2). However, Figures 5 and 6 clearly indicate almost the same intensity of sulfur peaks in the matrix regions (part 


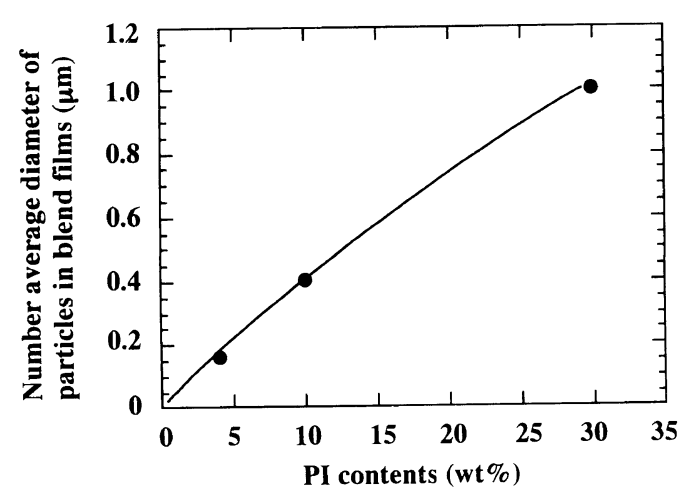

Figure 4. Relationship between PII content and number average diameter of the domain region in blend films.

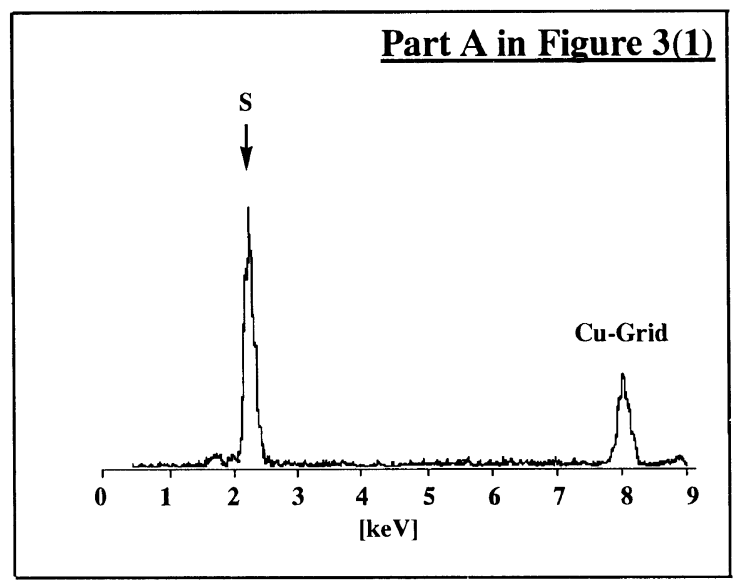

Figure 5. EDX analysis of blend film (PI/PES, 4/96) (part A in Figure $3(1))$.

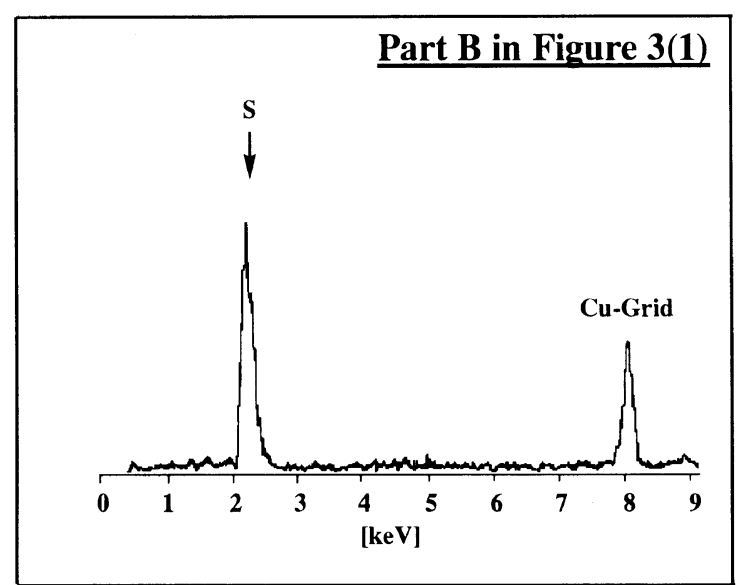

Figure 6. EDX analysis of blend film (PI/PES, 4/96) (part B in Figure $3(1))$.

A) and domain (part B) in Figure 3(1). There is thus an interpenetrating structure at the interfacial region between PI and PES, during composite film formation.

From these results, it can be suggested that composites having slight amounts of PI have a partially compatible phase, probably due to formation of an interfacial compatible region between PI and PES. Thus far, the blend film of rigid-rod PI and PES is considered incompatible and provides only phase separated cloudy films from the solution of PES and PII as a rigid-rod PI-precursor. The compatibility of PI and PES was 344

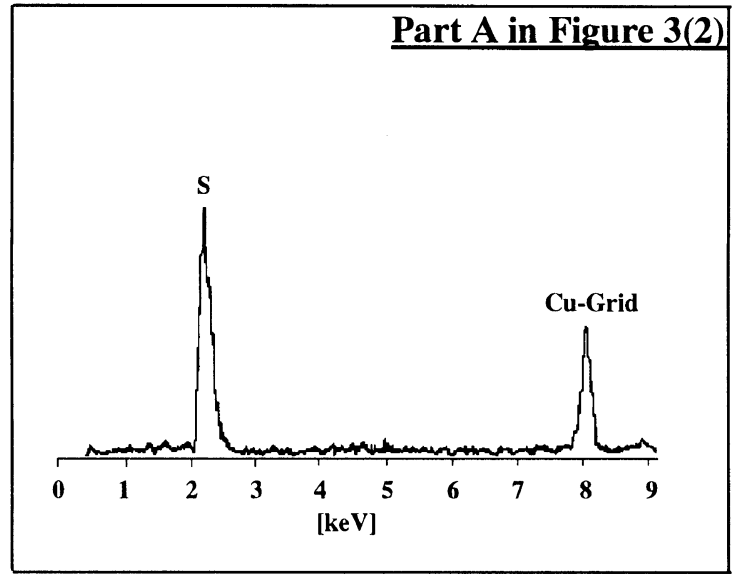

Figure 7. EDX analysis of blend film (PI/PES, 10/90) (part A in Figure 3(2)).

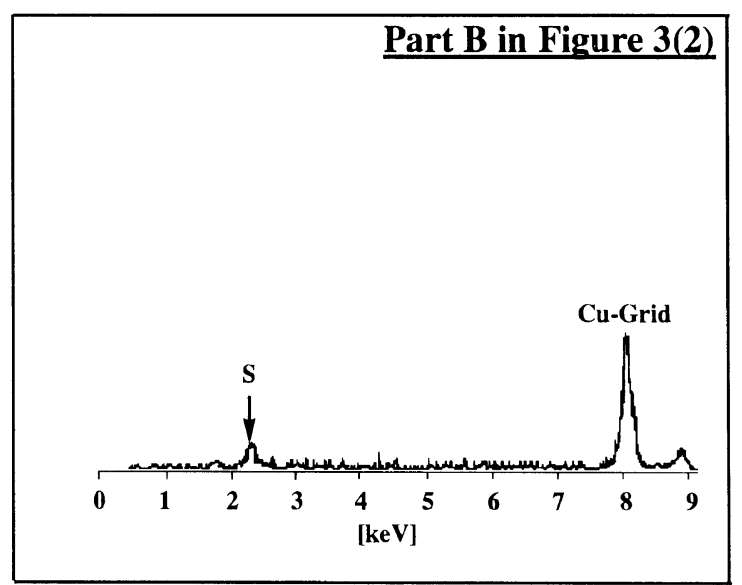

Figure 8. EDX analysis of blend film (PI/PES, 10/90) (part B in Figure 3(2)).

studied by morphological analysis such as polarizing microscope, TEM, and EDX. Polarizing microscopic observation made it clear that at lower PI the blend films were transparent and flexible. PI(TMBZ/PMDA) and PES were slightly compatible in the interfacial region and provided transparent flexible films having the phaseseparated structure consisting of PI domain well dispersed in the matrix PES.

\section{CONCLUSION}

The new PII as a rigid-rod PI-precursor was synthesized from PMDA and TMBZ. PII was soluble in aprotic polar solvents such as DMAc and was isomerized to the corresponding rigid-rod insoluble PI during film formation. The miscible solution blend of PII and PES was formed in DMAc. Blend film based on PI(a) and PES was produced by thermally induced isomerization of PII in the PES as a matrix polymer. Furthermore, transparent composite films with PI content of 2 to $10 \mathrm{wt} \%$ and micro domains of rigid-rod PI thermally generated in PES were obtained.

Acknowledgments. The authors are indebted to Hitoshi Nagasawa and Sadao Kato for their technical assistance, Takeyoshi Takahashi for performing the 
elemental analyses, and Hajime Tsuchiya of Nitto Technical Research Center for their TEM and EDX analysis. We also wish acknowledge financial support by a Grant-in-Aid for Scientific Research (No. 05453140) from the ministry of Education, Science, and Culture of Japan.

\section{REFERENCES}

1. T. E. Attwood, D. A. Barr, T. King, A. B. Newton, and J. B. Rose, Polymer, 18, 359 (1977)

2. R. N. Johnson, A. G. Farnham, R. A. Clending, W. F. Hale, and C. N. Merriam, J. Polym. Sci., A-1, 5, 2375 (1967).

3. H. Zeng and K. Mai, Makromol. Chem., 187, 1787 (1986).

4. X. Zhang and Y. Wang, Polymer, 30, 44 (1990).

5. S. Nakata, M. Kakimoto, and Y. Imai, Polym. J., 20, 80 (1990).

6. R. Yokota, R. Horiuchi, M. Kochi, H. Soma, and I. Mita, J.
Polym. Sci., C, Polym. Lett., 26, 215 (1988).

7. A. Morikawa, Y. Iyoku, M. Kakimoto, and Y. Imai, Polym. J., 24, 107 (1992).

8. N. Ogata, K. Sanui, and H. Itaya, Polym. J., 22, 85 (1990).

9. K. Sanui, Y. Kiyohara, M. Rikukawa, and N. Ogata, Reac. Funct. Polym., 30, 293 (1996)

10. J. S. Wallace, L-S. Tan, and F. E. Arnold, Polymer, 31, 2411 (1990).

11. K. C. Chuang, High Perform. Polym., 7, 81 (1995).

12. A. Mochizuki, T. Teranishi, and M. Ueda, Polym. J., 26, 315 (1994).

13. R. B. Carline, J. Am. Chem. Soc., 67, 928 (1945).

14. H-W. Schmidt and D. Guo, Makromol. Chem., 189, 2029 (1988).

15. T. Matsuura, Y. Hasuda, S. Nishi, and N. Yamada, Macromolecules, 24, 5001 (1991).

16. S. E. Delos, R. K. Schellenberg, J. E. Smedley, and D. E. Kranbuehl, J. Appl. Polym. Sci., 27, 4295 (1982).

17. A. Mochizuki, T. Teranishi, and M. Ueda, Macromolecules, 28, 365 (1995). 